\title{
Face Recognition based on Gabor Transform Combined with LBP
}

\author{
Jiyuan Wang ${ }^{1}$ and Eung-Joo Lee ${ }^{2 *}$ \\ ${ }^{1,2}$ Department of Information Communications and Engineering, University of \\ Tongmyong, Busan, 608-711 Korea \\ 1'yuan212108@naver.com, ${ }^{2 *}$ ejlee@tu.ac.kr
}

\begin{abstract}
Traditional human feature extraction algorithm based on Gabor transform folds the human face image and multiple scales and multiple direction kernel function for Gabor to obtain Gabor human feature with sampling and cascade. The obtained Gabor feature dimension of human face is high, the recognition process wastes time rather without rotation invariance, and performance of human face figure decreases at plane rotation. LBP is used in operator for texture analysis due to algorithm thought is sample, computation complexity is low and discernment is strong, etc. The above-mentioned analyzed and used widely for several years, LBP operator has fortissimo gray invariance and rotation invariance to overcome the problems of rotation shifting and uneven illumination. This dissertation adopts feature extraction method for combination of Gabor wavelet and LBP to research human face recognition to put forward to LBP algorithm with uniform pattern for improving the accuracy of human face feature recognition and flexibility of practical operation. The dissertation provides optimization selection and integration for human face feature extracted by Gabor wavelet and LBP to put forward to improved algorithm: feature extraction methods combined with $2 D$-Gabor wavelet and uniform LBP. This dissertation algorithm makes progress to improve recognition rate and decrease data redundancy with flexibility and effectiveness for human face recognition.
\end{abstract}

Keywords: Face recognition, Face feature extraction, 2D-Gabor wavelet, Local Binary Pattern(LBP), Uniform patterns LBP

\section{Introduction}

With the development of modern intelligent information technology, computer and Internet technologies are becoming increasingly developed. In the meantime, people face increasingly strong challenges in information security. In this context, a problem that needs to be solved urgently by the society is about how to identify and affirm identity accurately and fast [1]. At present, personal document or password is the principal method for identity authentication. As is known from long-term practices, the loss of personal documents and passwords has severely threatened the information security of people's daily life and work. In order to find an identity authentication method with higher security, researchers apply biological characteristics to identity authentication through the feature that human characteristics cannot be copied. Subsequently, the method of indentifying biological characteristics enjoys fast development and application. Compared with the traditionally used methods of identifying passwords and personal documents, the biological characteristic recognition has numerous advantages, including being fast, safe, un-duplicated and difficult to be forged [2]. As is known to all, biological characteristics (such as fingerprints, genes, profiles and voices) are characteristics unique to a person. According to such biological characteristics, numerous technical means have been

Received (August 19, 2017), Review Result (November 5, 2017), Accepted (November 19, 2017)

* Corresponding Author 
developed and designed based on computer fundamentals in the current phase to identify the human identity, including voice recognition. Currently, the recognition technology in this regard has been widely accepted and applied [3]. In the above-mentioned human characteristic recognition technology, the facial recognition technology receives unanimous acclaims for more friendly interactions and no psychological barrier in use. Secondly, the analysis of human facial expressions helps people to obtain the information that cannot be identified by other methods [4]. In addition, the biological characteristic recognition has several advantages, such as being non-contact, non-mandatory and noninvasive. Moreover, the human face triggers memories more easily than other body parts. Hence the biological characteristic recognition is of great significance to research the facial recognition technology. That's why it is the current hot-spot direction of identity authentication, image processing and other subjects [5].

The exploration of facial recognition technology is of important scientific values, which are represented in two aspects: Firstly, the research on the facial recognition technology deepens people's understandings of humans' visual system; secondly, the research on the facial recognition technology provides a basis for applying artificial intelligence. Hence setting up a facial recognition system on the computer has significant application values and application prospects for identifying facial images [6].

At present, the research priorities of the facial recognition include the methods of classifying images and feature abstraction algorithm. The latter is the key and plays the decisive role in determining the eventual effect of facial recognition. The Gabor transform is surprisingly similar to the receptive model of humans' retina cells. It is thus capable of obtaining the least uncertainty in frequency and time. Moreover, it has strong robustness targeting at external interferences, such as sunshine, postures and expressions. The traditional facial feature abstraction methods, which are based on Gabor transform, firstly execute the convolution of facial expressions to produce the multi-direction and multiscale kernel function of Gabor transform. Secondly, the cascading and downward sampling are conducted to obtain Gabor facial features. Such a method yields a high number of dimensions, which requires much time and does not need the rotational invariance. The recognition performance decreases as the facial image rotates on a surface. The LBP (Local Binary Pattern) is an operator that analyzes patterns. With a simple algorithm, the calculation process has several advantages. For instance, it is relatively simple and has a strong distinguishing capacity. In recent years, the LBP operator has been widely researched and applied. With a strong gray invariance and rotational invariance, the LBP operator is capable of decreasing the impact of low rotational displacement and uneven sunshine.

Based on the above-mentioned problems, the thesis abstracts features by combining the Gabor wavelet and LBP. It then researches the facial recognition and proposes the uniform pattern LBP. In order to improve the accuracy of abstracted facial features and the flexibility of actual utilization, the thesis carries out an optimized selection and integration of facial features abstracted through Gabor wavelet and LBP. Meanwhile, it proposes an improved algorithm: namely the feature abstraction algorithm that combines 2D-Gabor wavelet and uniform pattern LBP. The thesis improves the recognition rate and reduces data redundancy effectively. Moreover, it is applicable to common facial bases, which effectively identifies facial expressions.

\section{Related Work}

\subsection{Introduction to Gabor Wavelet Transform}

Gabor wavelet transform has the visual characteristics of living organisms, which are extremely similar to the visual stimulus responses of human's biological visual system. However, such characteristics are only similar to simple visual cells. In addition, the 
Gabor wavelet transform also has several advantages. Its best characteristic is giving equal considerations to the information in the time domain. However, the Gabor wavelet transform cannot set up an orthogonal basis unless under certain parameters [7]. The Gabor wavelet has significant characteristics, including direction selection, scale selection characteristics and the sensitivity to image margin, which make people fond of applying it to the visual and information understanding fields. The 1-D Gabor wavelet has various excellent qualities. Subsequently, researchers propose the 2-D Gabor wavelet transform. Compared with the 1-D Gabor wavelet, the 2-D wavelet does not need to change the column vector to identify faces. However, the 2-D Gabor wavelet has all the advantages owned by the 1-D Gabor wavelet transform. It is thus frequently applied in image processing and model recognition [8].

Gabor wavelet transform has significant characteristics in technology and biology. That's why the Gabor wavelet is adopted in the image pre-treatment.

Firstly, the Gabor wavelet kernel function is capable of eliminating direct current components, namely eliminating $\exp \left(-\frac{\sigma^{2}}{2}\right)$ in the attached plane wave. The Gabor wavelet is not restricted by the changes of light on the facial image. Generally speaking, the Gabor Wavelet is applied in the tests that place high requirements on light.

Secondly, the Gabor wavelet kernel function allows the user to adjust the scales and directions of Gabor wave filter parameters. In the meantime, the small-scale filtering can reflect the local structure. If the filtrating has a large scale, it is capable of reflecting the whole structure. Furthermore, the Gabor wavelet filtration can represent the information related to the gray distribution of images in all directions and scales.

\subsection{D-Gabor Wavelet Transform Principle}

The Gabor wavelet is frequently used in multi-resolution and multi-channel analyses, which are similar to humans' visual characteristics. In the 1980s, Daugman transformed Gabor wavelet into 2-D model and constructs 2-D Gabor wavelet. This is because the minimum uncertainty of time and frequency is obtained through 2-D Gabor. In this regard, it is similar to the receptive model of humans' retinas [9]. Hence the Gabor wavelet algorithm is the best characteristic abstraction algorithm. In the thesis, the utilization of the 2-D Gabor wavelet in the Gabor transform is the research priority.

Here is the expression for the two-dimensional Gabor wavelet kernel function:

$\Psi_{\text {uv }}=\frac{\left\|k_{u v}\right\|^{2}}{\sigma^{2}} \mathrm{e}^{-\left(\left\|k_{u W}\right\|^{2}\|z\|^{2} / 2 \sigma^{2}\right)}\left[\mathrm{e}^{i z k_{u v}}-\mathrm{e}^{-\left(\sigma^{2} / 2\right)}\right]$

In this function $\mathrm{u}$ and $\mathrm{v}$ as parameters. Where: $\mathrm{u}, \mathrm{v}$ is respectively express the direction and scale factor; $\mathrm{Z}=(\mathrm{x}, \mathrm{y})$ is the input of the space coordinates of the image, the twodimensional coordinate is $\mathrm{x}, \mathrm{y}$.

The constants associated with wavelet frequency bandwidth are represented by $\sigma$ and can be obtained by the following formula:

$$
\sigma=\sqrt{2 \ln 2}\left(\frac{2^{\varphi}+1}{2^{\varphi}-1}\right)
$$

The relationship between the width of the window and the wavelength are determined by the above equation. The half - peak bandwidth of the octave is represented by $\varphi$. The center frequency of filter shown as $\mathrm{k}_{\mathrm{uv}}$

$$
\mathrm{k}_{\mathrm{uv}}=\left(\begin{array}{l}
\mathrm{k}_{\mathrm{x}} \\
\mathrm{k}_{\mathrm{y}}
\end{array}\right)=\left[\begin{array}{l}
\mathrm{k}_{\mathrm{v}} \cos \phi_{\mathrm{u}} \\
\mathrm{k}_{\mathrm{v}} \sin \phi_{\mathrm{u}}
\end{array}\right]
$$


Get command of the width of Gauss window, the wavelength and the direction of the oscillation. Where the $\emptyset_{\mathrm{u}}$ is shown as texture direction, and reflected on filter direction selectivity; $\mathrm{k}_{\mathrm{v}}=\mathrm{k}_{\max } / \mathrm{f}^{\mathrm{v}}$ is shown as texture wavelength, the maximum center frequency is shown as $\mathrm{k}_{\max }$, in the frequency domain the filter is shown as $\mathrm{f}^{\mathrm{v}}$

Through Euler formula, cosine and sine waves represent the real and imaginary parts of the two-dimensional Gabor wavelet

$$
\begin{aligned}
& \operatorname{Re}\left(\psi_{\mathrm{uv}}\right)=\frac{\left\|\mathrm{k}_{\mathrm{uv}}\right\|^{\mathrm{z}}}{\sigma^{2}} \mathrm{e}^{-\left(\frac{\left\|\mathrm{k}_{\mathrm{uv}}\right\|^{2}\|z\|^{2}}{\mathrm{zv}^{2}}\right)}\left[\cos \left(\mathrm{zk}_{\mathrm{uv}}\right)-\mathrm{e}^{-\left(\sigma^{2} / 2\right)}\right] \\
& \operatorname{Im}\left(\psi_{\mathrm{uv}}\right)=\frac{\left\|\mathrm{k}_{\mathrm{uv}}\right\|^{2}}{\sigma^{2}} \mathrm{e}^{-\left(\frac{\left\|\mathrm{kuv}_{\mathrm{uv}}\right\|^{2}\|z\|^{2}}{\mathrm{vv}^{2}}\right)} \sin \left(\mathrm{zk}_{\mathrm{uv}}\right)
\end{aligned}
$$

The properties around a pixel point in the image can be represented by a twodimensional Gabor wavelet. The filtering process of the image is completed during the convolution of the Gabor function and the image

The parameters of filter should be selected according to physiological and psychological experiments. the researchers validated through experiments for the following conclusions, when the filer bandwidth $\phi$ is 0.5 octave, $\mathrm{k}_{\max }=\frac{\pi}{2}$ is one of the biggest center frequency, if the image size is $128 \times 128$, we can get the best experimental data. Follow the function below, if $\phi=0.5$, the $\sigma \approx 2 \pi \circ$ So, in this paper we choose filter is $\mathrm{k}_{\max }=\frac{\pi}{2}, \sigma \approx 2 \pi$ 。

$$
\sum=\frac{1}{\mathrm{M}} \sum_{\mathrm{i}=0}^{\mathrm{M}-1}\left(\chi_{\mathrm{i}}-\mu\right)\left(\chi_{\mathrm{i}}-\mu\right)^{\mathrm{T}}
$$

\subsection{Face feature extraction based on Gabor Transform}

Through the Gabor transform for a face image, we can get the Gabor kernel function $\Psi_{v}$ and face image convolution I(z), the following is a specific said:

$$
W_{\mathrm{uv}}(\mathrm{z})=\mathrm{I}(\mathrm{z}) * \psi_{\mathrm{uz}}(\mathrm{z})
$$

In the function above: different parameters $\mathrm{u}$ and $\mathrm{v}$ corresponding Gabor base through bits of $\Psi_{\mathrm{uv}}(\mathrm{z})$, the $W_{\mathrm{uz}}(\mathrm{z})$ is shown as Gabor base and the Gabor characteristics which is the image $I(z)$ after the convolution, among them, $v \in\{0,1,2,3,4\}, u \in\{0,1,2,3,4,5,6,7\}$.

The real and imaginary part of the plural response generated by Gabor convolution [10], because in fact imaginary part is not through the smooth peak response, vibration formed around the image, is not easy to identify. The concrete as shown in the figure below:

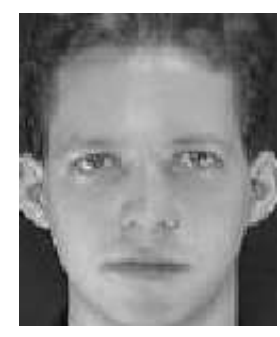

Figure 1. The Original Face Image 


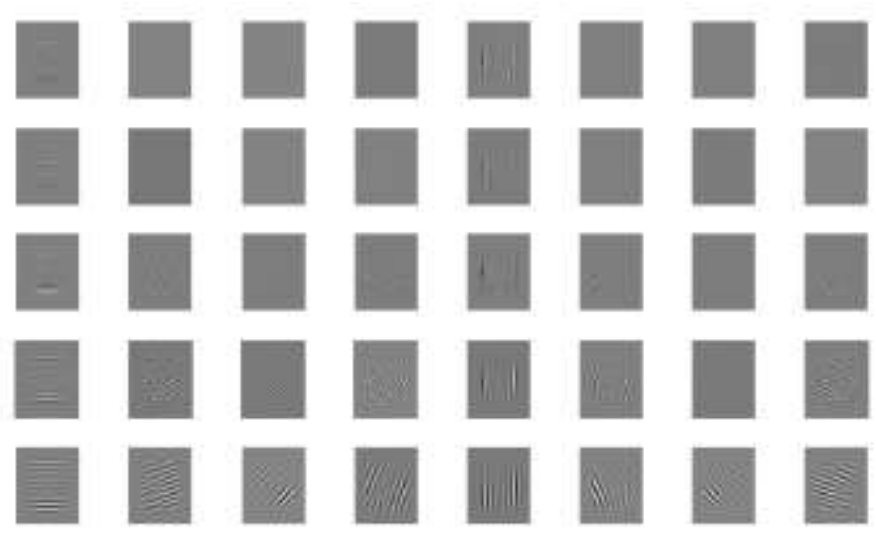

Figure 2. Gabor Real Response of the Face Image

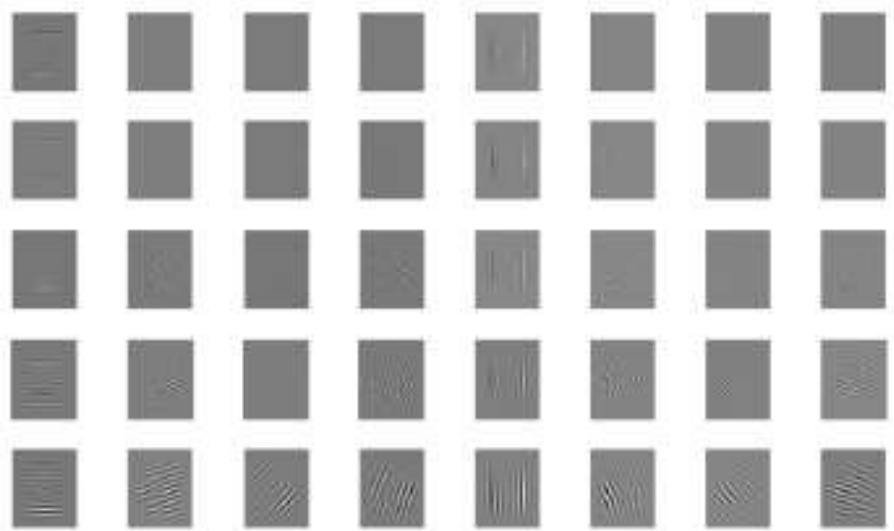

Figure 3. Gabor Imaginary Part Response of the Face Image

Local energy spectrum of the image through the reflection amplitude information is expressed, at the same time a better embodiment of smoothness is shown around the image, it can be more convenient to identify, and this article said Gabor transform response of the face image is expressed by amplitude characteristics of the Gabor transform.

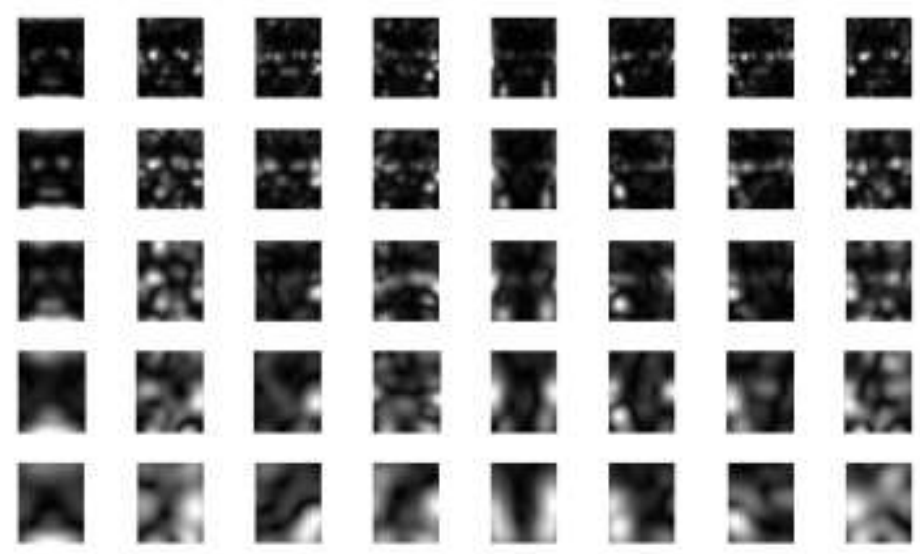

Figure 4. Gabor Amplitude Characteristics of Face Image 
After the Gabor feature is obtained, linked the 40 eigen values of Gabor, and the Gabor characteristics of the face image can be obtained. The following is the cascade process:

First of all, a cascade of image position (x, y) in the corresponding 40 Gabor feature $\mathrm{O}$ $(\mathrm{x}, \mathrm{y})$, we can obtain:

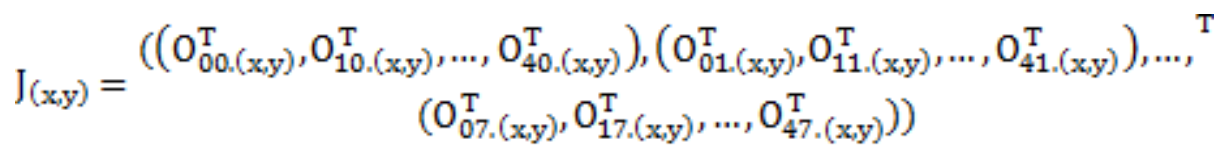

Secondly, the whole pixel position of the cascade image $(x, y) \in I$ is the $J(x, y)$ of the image, and the Gabor feature vector of the image can be obtained below:

$$
J=\left\{J_{(x, y)} \mid(x, y) \in I\right\}
$$

From the above, the Gabor image feature dimension is 40 times of the original image dimension, which is difficult to classify and identify such images with high redundancy. So, reduce the dimensions of the Gabor feature is necessary.

\section{Local Binary Pattern Principle}

\subsection{LBP Operator}

LBP is an effectively describe operator within the scope of the gray scale of texture, the original operator area is $3 * 3$, the window is mainly composed of a center pixel $g_{c}$ and 8 adjacent pixels $g_{0}, \cdots, g_{7}$. In LBP operator, the texture $T$ of the image can be denoted as [11]:

$$
\mathrm{T} \approx \mathrm{t}\left(\mathrm{g}_{0}-\mathrm{g}_{\mathrm{c}}, \cdots, \mathrm{g}_{7}-\mathrm{g}_{\mathrm{c}}\right)
$$

Will in the window within another 8 pixels to center pixel as a benchmark for binarization operation, concrete process is: the center pixel as the threshold, will the other 8 pixel gray value compared with the threshold, assumptions surrounding pixel size is less than the center pixel, so the pixel tag to 0 , or 1 . After the binary operation, the new $\mathrm{T}$ calculation formula is obtained:

$$
\mathrm{T} \approx \mathrm{t}\left(\mathrm{s}\left(\mathrm{g}_{0}-\mathrm{g}_{\mathrm{c}}\right), \cdots, \mathrm{s}\left(\mathrm{g}_{7}-\mathrm{g}_{\mathrm{c}}\right)\right)
$$

The operation of the binary is expressed as:

$$
S(x)= \begin{cases}1 & x \geq 0 \\ 0 & x<0\end{cases}
$$

Complete formula (12) after operation, eight neighboring pixels in a $3 * 3$ window can be corresponding to the eight binary values, then use the calculation formula (13) weighted summation of pixel location, to calculate the area of LBP values.

$$
\operatorname{LBP}_{\left(\mathrm{x}_{\mathrm{c}} \mathrm{y}_{\mathrm{c}}\right)}=\sum_{\mathrm{i}=0}^{7} \mathrm{~s}\left(\mathrm{~g}_{\mathrm{i}}-\mathrm{g}_{\mathrm{c}}\right) 2^{\mathrm{i}}
$$

The LBP operator is adopted to calculate the pixels in images and obtain the LBP values that correspond to different pixel positions. In this way, the pattern information of an image is described. The early LBP operator is derived from the locally-nearest concept of pattern. In the early phase, its function is to assist the measurement of the comparison of the local region, namely LBP/C. Figure 4 describes the calculation methods of the early 
LBP in the early phase in details. The comparative component $\mathrm{C}$ is the mean value of all pixel points in the field that are $\geq$ central pixel points $\mathrm{g}_{\mathrm{c}}$ and all pixels within the region that are <central pixel points $\mathrm{g}_{\mathrm{c}}$.

The basic LBP operator has the defect of only covering an extremely small region within the radius. It is thus unable to meet the demands of different sizes and frequency patterns [12]. Ojala et al. conducted the improvement research to make up for the abovementioned defects, meet the demands for various sizes and meet the requirements of grayness and rotational invariance.

\subsection{Uniform Pattern LBP}

The value of $\mathrm{LBP}$ coding changes as the number of sampling points varies. $\mathrm{LBP}_{\mathrm{P}_{\mathrm{R}} \mathrm{R}}$ can form $2^{\mathrm{P}}$ models. The typical $\mathrm{P}$ value is usually 8 . Hence the traditional LBP coding has 256 modes. With the application of LBP coding mode, it is discovered the abovementioned coding modes restrict the descriptions of patterns to some extent. For instance, 16 sampling points are selected in the domain of $4 * 4$, which can form $224=16,777,216$ modes. According to relevant practices, partial modes are unfavorable or do no good for the pattern description. As is shown by subsequent practical research, the adoption of an excessive large number of sampling points will result in excessive modes. Meanwhile, the excessive number of binary mode types faces the following defects in information abstraction: Firstly, the calculation amount is both huge and complex, requires high costs and needs a large storage space, which is all unfavorable for abstracting the patterns of images. Secondly, the produced histograms are scarce, which is unfavorable for abstracting image characteristics and describing characteristics. Given considerations to the above-mentioned defects, the operator needs to lower the dimensions of characteristics abstracted by the LBP operator, which is favorable for obtaining more perfect pattern information and describing characteristics more detailed [13].

There is a large number of LBP operator modes, which have certain defects in abstracting pattern characteristics. Hence researchers start to improve traditional operators. Based on the traditional LBP algorithm, Ojala et al. proposed the uniform pattern LBP. The uniform patterns refer to the LBP modes that have a higher efficiency. All the modes except the uniform pattern are mix modes [14][15]. The characteristic of the uniform pattern is: The binary sequence does not have excessive jumps, which jumps from 1 to 0 or from 0 to 1 and do not exceed 2. According to the above-mentioned characteristics, Ojala defined the uniform pattern, namely it is the mode in which the binary sequence jumps from 1 to 0 or from 0 to 1 in the LBP operator. The binary system of 0 or 1 is directly related to the binary system. Furthermore, Ojala also proposed the method of testing the uniform pattern and made judgment according to the uniform variable $\mathrm{U}$. The calculation formula is as follows:

$$
\mathrm{U}\left(\mathrm{LBP}_{\mathrm{p}, \mathrm{R}}\right)=\sum_{\mathrm{p}=1}^{\mathrm{P}}\left|\mathrm{s}\left(\mathrm{g}_{\mathrm{p}}-\mathrm{g}_{\mathrm{c}}\right)-\mathrm{s}\left(\mathrm{g}_{\mathrm{p}-1}-\mathrm{g}_{\mathrm{c}}\right)\right|
$$

The calculation process of the above-mentioned formulas is about comparing the number of digits in the binary system and the number of the sequence after moving one digit. If the calculation results meet $\mathrm{U} \leq 2$, it is a uniform pattern. Otherwise, it is classified as a mixed mode. In the specific calculation, both the uniform pattern and the common mode conduct mapping through searching. The inquiry table of $\mathrm{LBP}_{\mathrm{B}_{3} 1}^{\mathrm{u2}}$ is shown in Table 3.1. It means the Uniform Pattern LBP selects $\mathrm{P}$ sampling points within the radius of $R$. The maximum value of $U$ is 2 . The superscript $u 2$ represents the LBP operator is a uniform pattern. Fig.5 describes the uniform pattern of $\mathrm{LBP}_{\mathrm{8}, 1}^{\mathrm{u2}}$, whose field radius is 1 and has 8 sampling points. 


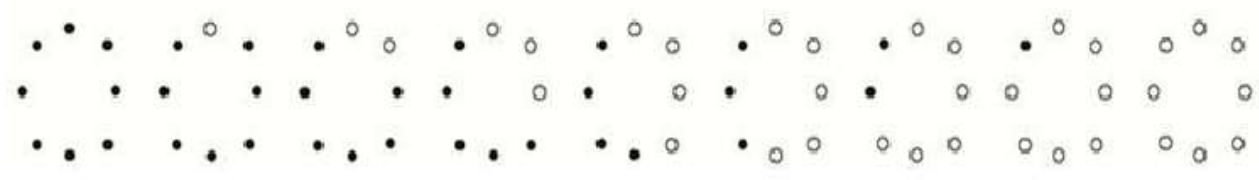

Figure 5. The uniform pattern part of the $\operatorname{LBP}(8,1)$

The uniform pattern mostly adopts the LBP algorithm, which effectively improves the phenomenon of excessive binary modes and reduces the number of models from $2 p$ to $P$ $(\mathrm{p}-1)+2$. In the process of histogram statistics, the number of vectors in the histogram will change as the LBP algorithm varies. If a facial expression image passes the LBP operator, the histogram has 256 dimensions. In the process of calculating histogram through the LBP8, 1 of the uniform pattern, there are only 58 modes. Each uniform pattern corresponds to an independent histogram gathering box. If all non-uniform patterns in the input are eliminated, the image will lose its micro-mode structure, which is because the facial recognition takes the non-uniform pattern as the main judgment basis. It is thus necessary to combine two modes organically and represent the facial characteristics. After taking all non-uniform patterns into the histogram gathering box as the same category, there are 59 dimensions in all.

In the following, the advantages of the uniform pattern LBP operator are described in details: Firstly, the calculation is easy and simple, which substantially reduces the calculation of characteristic values and characteristic vectors, improves the calculation efficiency and reduces calculation expenditures. Here the abstracted pattern characteristic information is represented through a histogram. Secondly, the characteristic is strongly distinguishable. Hence adopting the Uniform Pattern LBP for calculation is capable of representing data information, such as the dark points and shiny points in the image [16]. Meanwhile, the Uniform Pattern LBP represents the correlations and functions of pixels and other surrounding pixel points to further describe the pattern information of an image. Secondly, the algorithm also has high robustness targeting at the rotational invariance and the changes to light and posture changes [17][18]. Thirdly, no training is needed before using the algorithm. The commonly adopted facial recognition technology needs to set up a facial information training mode. If there are huge differences between the samples of the training set and tested samples, it will influence the recognition rate. Conversely, the LBP operator adopted by the uniform pattern does not need to prepare the facial information training. Meanwhile, the calculation results are characteristic vectors of a histogram, which are highly practical.

The LBP algorithm represents image grayness through coding. Hence the final result is still an image after adopting this method for image processing. Before and after the processing, there are certain differences between the grayness of pixels in the image. The author thus divides the images after adopting the mean mode LBP coding. Figure 6 is the image case after the Uniform Pattern LBP is divided into $3 * 3,4 * 4,5 * 3$ and $6 * 5$.

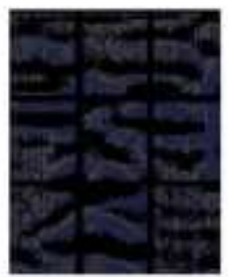

$3 * 3$

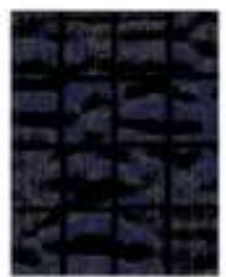

$4 * 4$

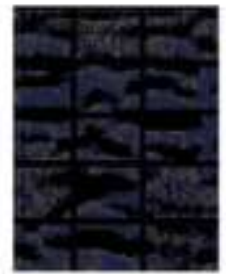

$5=3$

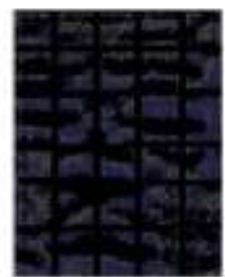

$6 * 5$

Figure 6. Example of image by different blocking after dealing with the Gabor and LBP 


\section{2D-Gabor Wavelet and Uniform Pattern LBP}

\subsection{Introduction to the Algorithm of Combing 2D-Gabor Wavelet and Uniform Pattern LBP}

In the process of researching the facial recognition process, the characteristic abstraction method plays a major and crucial role. At present, the key of researching facial recognition technology is to find a characteristic abstraction method reflects the overall characteristic information of image and gives equal considerations to the local characteristic information of the image. The lack of information related to the detailed image information lowers the rate of indentifying abstracted characteristics. However, excessive detailed information increases the dimension of images. Moreover, it causes the sample scale to be large and excessively scattered. The undistinguished boundaries between each category make effective characteristic classification impossible. Hence the thesis adopts the 2D-Gabor wavelet to abstract characteristics from primitive images, including abstracting multi-perspective characteristics and multi-scale characteristics to lower the inference of illumination and rotation on the image abstraction. The LBP algorithm is capable of conducting coding and setting up a histogram. It thus makes adequate representation of detailed image information. The PCA method lowers the number of image pixels.

In the LBP calculation process, the grayness comparison is adopted to select the final abstracted value. Two codes that are close to each other are correlated to each other and will be affected by the noise interference. If the 2D-Gabor wavelet abstracts features from the image, it is capable of overcoming this problem. By filtering the image through 2DGabor wavelet, the convolution of facial images at least takes two channels, which make the abstracted detail characteristics more perfect and useful. Meanwhile, it enhances the ability of avoiding interferences for the image.

The 2-D Gabor wavelet not only enhances the anti-inference capacity, but my also abstracts the characteristic information of several scales and directions. Targeting at the abstraction of local patterns, the LBP algorithm represents excellent local characteristics of an image. In the meantime, it helps to reduce the number of dimensions effectively. After adopting the 2D-Gabor wavelet to filter images, the user may use the LBP algorithm to code for it, which makes up for the defects of Gabor wavelet abstraction algorithm effectively.

\subsection{D-Gabor wavelet and Uniform Pattern LBP Combining Algorithm Illustration}

Due to the 2D-Gabor can be regarded as a two-dimensional Gaussian function modulation of plural sine fence, Gaussian filter method as a kind of low pass filter and can increase the proportion of LBP and unified model, so it can improve the stability and accuracy of feature extraction. The algorithm combines the advantages of Gabor wavelet and LBP operator to achieve better results.

In this paper, a feature extraction method based on 2D-Gabor wavelet and uniform pattern LBP is selected to briefly introduce the extraction process:

(1) Enter a face image size of $112 * 92$ pixels.

(2) Using the image and 40 different directions and sizes of Gabor filter equation to convolve processing, we can obtain 40 images, the dimensions are unchanged, and the size is still $112 * 92$ pixels, and one of them is shown in Figure 8

(3) After the convolution of the image and then doing the uniform LBP codes, block model is selected for $5 * 3$, which is shown in Figure 9, we can get a list of histogram, shown in Figure 10. 
(4) Integrating all 40 histogram, doing sequence of processing for the 40 histogram, which is shown in Figure 11, then use PCA to reduce the dimension and get the result.

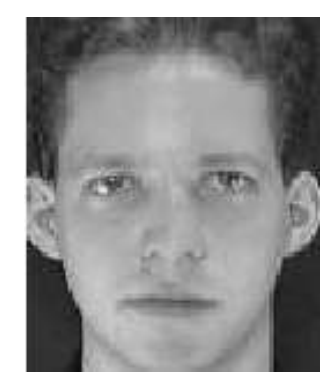

Figure 7. The Original Face Image

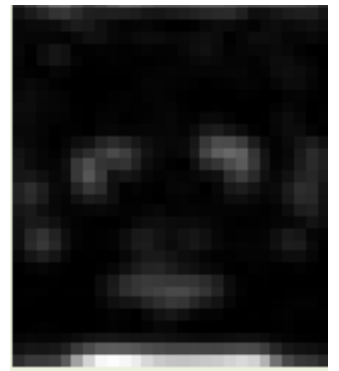

Figure 8. The Processed Image by Gabor

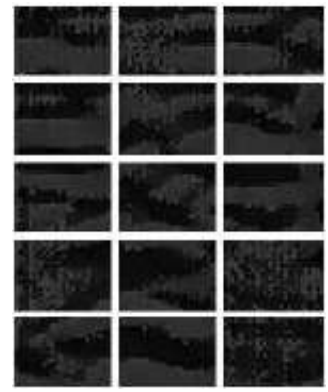

Figure 9. The Image of LBP by Block Partition Modes is $5 \star 3$

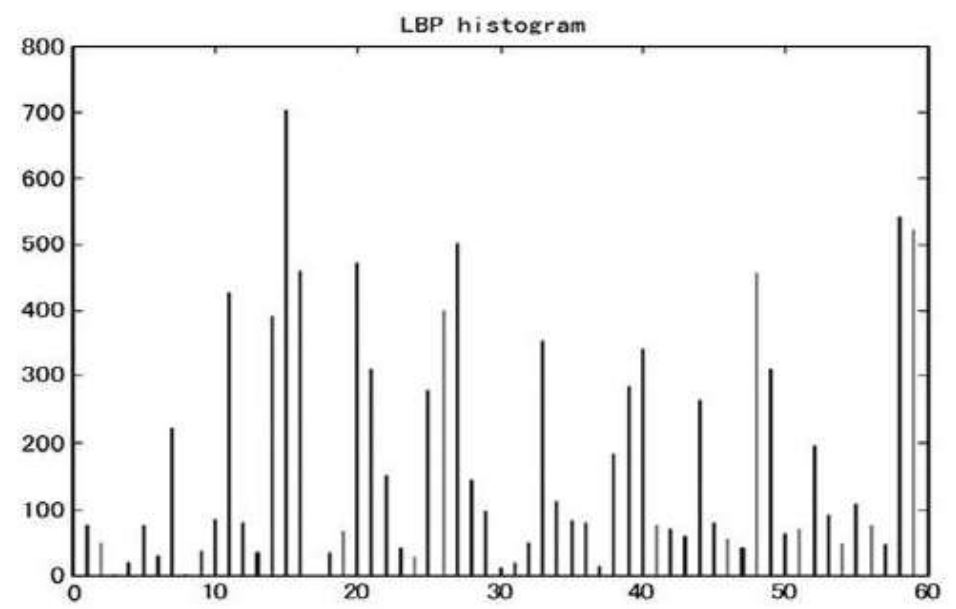

Figure 10. Histogram of one-node LBP feature 


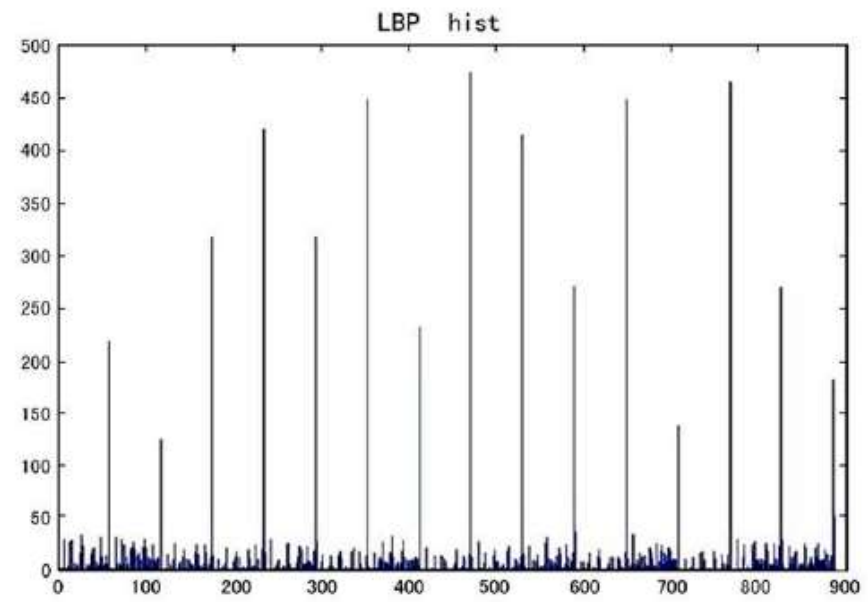

Figure 11. Histogram of the Union Feature by Blocking LBP

\section{Experimental and Analysis}

The former section describes the facial recognition algorithm of 2D-Gabor wavelet and uniform pattern LBP. It also describes and experiments the algorithm procedure. In the following part, the thesis researches the size of the influences of the kernel function window of the 2D-Gabor wavelet and the LBP division model on the recognition rate. Meanwhile, relevant experiment research is adopted to test and prove the effect of using the 2D-Gabor and LBP uniform pattern in identifying facial expressions, as well as the feasibility and validity of applying this method in identifying facial expressions. Furthermore, the thesis compares with other common methods to test the feasibility and validity of this method.

\subsection{Impact of 2D-Gabor Kernel Size on Recognition Rate}

The Formula (1) is the $2 \mathrm{D}-$ Gabor wavelet calculation equation. In the equation, $\mathrm{Z}=(\mathrm{x}$, $\mathrm{y})$ : it is the coordinate of the characteristic image that corresponds to the pixel space. The convolution varies as $(\mathrm{x}, \mathrm{y})$ changes. Hence the domain of the Gabor wavelet kernel window is the range of $(\mathrm{x}, \mathrm{y})$. Whether the window is asymmetrical is related to the characteristic abstraction effect of 2D-Gabor and the shape of the wave filter. If the kernel windows are asymmetrical, the non-zero part of Gabor is basically located in the center of the Gabor wave filtration device. If the kernel windows are asymmetrical, the non-zero frequency spectrum will be farther away from the Gabor wave filtration device center as the window increases, which makes it unfavorable for abstracting window images. The thesis expounds on the role of kernel window to the facial recognition system. The asymmetrical windows are not discussed further.

In the research process, the thesis determines the functions of five asymmetrical 2DGabor wavelet kernel functions. Meanwhile, other parameters are designated as the fixed value to reduce the impact of other factors on experimental results. Five scales and eight directions are set. Meanwhile, $\varphi_{\mathrm{u}}=\frac{\pi \mathrm{u}}{4}, \sigma=\pi, \mathrm{k}_{\mathrm{v}}=\mathrm{k}_{\max } / \mathrm{f}^{\mathrm{v}}$, namely the biggest threshold value of frequency is $k \max =\pi / 2$; $\mathrm{f}$ is the interval of the frequency domain wave filtration device, whose value is $\mathrm{f}=\sqrt{2}$. The training sample and number of testing samples are both 200 sets. The 2D-Gabor wavelet and LBP uniform pattern are combined to abstract characteristics (the division model is $5 * 3$ ). The following table is about the relations between the feature dimensios and recognition rate. 


\section{Table 1. The Relationship between Different Windows from Gabor Kernel Function and Recognition Rates}

\begin{tabular}{|c|c|c|c|c|c|c|c|c|c|c|c|c|c|}
\hline \multirow[t]{2}{*}{ Size } & \multicolumn{13}{|c|}{ Recognition Rate(\%) } \\
\hline & 25 & 50 & 75 & 100 & 125 & 150 & 175 & 200 & 225 & 250 & 275 & 300 & Ave \\
\hline $7 * 7$ & 70.5 & 74.5 & 81.5 & 80.0 & 76.0 & 78.5 & 78.5 & 80.5 & 79.5 & 80.5 & 78.5 & 78.0 & 71.46 \\
\hline $19 * 19$ & 82.0 & 84.5 & 85.0 & 85.0 & 84.0 & 84.0 & 84.0 & 83.0 & 84.0 & 82.5 & 82.5 & 84.0 & 83.71 \\
\hline $35 * 35$ & 87.5 & 86.0 & 89.5 & 88.5 & 86.0 & 87.5 & 87.0 & 86.0 & 85.0 & 85.0 & 84.0 & 82.5 & 86.25 \\
\hline $51 * 51$ & 82.5 & 84.0 & 87.5 & 85.5 & 86.0 & 85.0 & 86.0 & 84.5 & 84.0 & 84.0 & 85.0 & 82.0 & 84.58 \\
\hline $65 * 65$ & 81.5 & 82.5 & 82.5 & 81.0 & 80.5 & 82.0 & 81.0 & 82.5 & 81.0 & 82.0 & 80.5 & 81.0 & 81.27 \\
\hline
\end{tabular}

As is shown in the following table, the algorithm recognition rate that corresponds to the kernel window of various sizes is higher than other dimension numbers when there are 75 dimensions. In other words, the recognition rate does not always increase as the number of dimensions increases under the kernel windows of different sizes. Instead, it presents the process of first increase and then decrease. When the window size is $35^{*} 35$, the recognition rate is the highest, which provides a reference basis for the following experiments.

\subsection{The Influence of Block Mode on Recognition Rate}

The recognition rate in this paper is affected by various factors, this section we discusses the LBP algorithm of different block model and the dimension change on the recognition rate and using Gabor transform to extract image features, then according to the block method to blocking. In the research we will respectively block in $6 * 5,5 * 3,4$ $* 4$ and $3 * 3$, and on the relationship between the block model and the algorithm of recognition is verified, the following experimental is setting: 2D-Gabor wavelet kernel window size is $35 * 35$, a total of five dimensions of eight directions, and the test set including 200 samples images, to accomplish the classification.

In the research below is talk about the characteristic dimension influence on recognition algorithm, the each image by the dimensions of the LBP feature extraction is 59 dimensions, after $3 * 3$ LBP block, the dimension increase by 531 dimension, the training image sample is 200 , so the experiment can be from 0 to 500 dimensions one by one to verify this dimension.

In the following Figure 12: when the number of samples is 200 , the blocking is $3 * 3$, the dimension is about 75 , the algorithm has the highest recognition rate, but the rate of recognition is the same as the increase of dimension. Therefore, in the analysis of the influence of the block mode of LBP on the recognition rate, the feature dimension of each face image is fixed to 75 dimensions

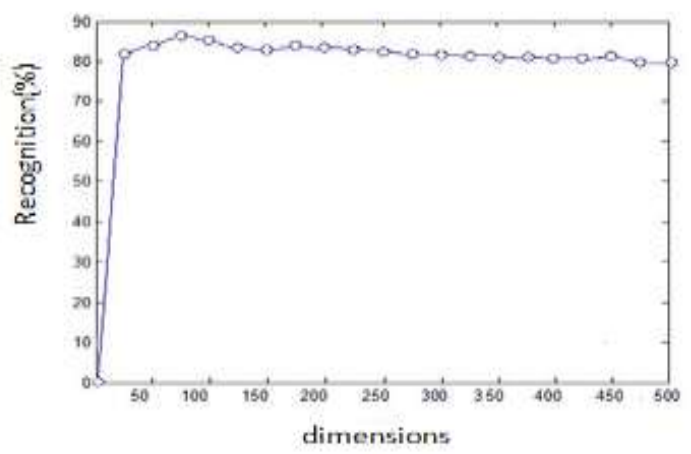

Figure 12. Relationship between the Character Dimension and Recognition Rates by Block Partition Modes is $3{ }^{\star} 3$ 
When the number of training samples is 200 the average highest average recognition rate of each block partition modes is in the below:

\section{Table 2. Highest Average Recognition Rates on Different Block Partition Modes by Training Sample is $\mathbf{2 0 0}$}

\begin{tabular}{c|c}
\hline Block Partition Modes & Recognition Rate(\%) \\
\hline $3 * 3$ & 83.74 \\
\hline $4 * 4$ & 84.63 \\
\hline $5 * 3$ & 87.14 \\
\hline $6 * 5$ & 85.72 \\
\hline
\end{tabular}

We can see from Table 2, when the LBP block pattern is $5 * 3$, the average recognition rate is highest, thus in running the entire algorithm we choose LBP block pattern for $5 * 3$, it laid the foundation for the following experiments.

\subsection{Impact of Different Feature Abstraction on the Recognition Rate}

In order to prove the practicability of algorithm in the thesis, this section compares the algorithm of the thesis and several common algorithms in the condition of consistent hardware and software. It also compares four different feature abstraction methods of LBP, Gabor PCA and 2D-Gabor wavelet and uniform pattern LBP (hereinafter abbreviated to 2D-Gabor+UPLBP). The method of combining LBP, Gabor and 2D-Gabor wavelet and LBP adopts PCA to reduce dimensions. In the experiment, Gabor selects five scales and eight directions, whose sizes are $35 * 55$. The particle partition mode of facial figures is $5^{*} 3$ and the number of dimensions is 75 dimensions. In the following, the experiment is carried out in ORL facial database to compare the recognition rate of four different algorithms in the same conditions.

The ORL database contains 400 samples with 40 groups, every groups of 10 , each group is the same person's different posture and expression, the size is $112 \times 92$. In ORL database randomly selected samples of training samples for experiment, when the sample data of 80-320, take 30 experiments, and calculate the average. The experimental results are shown in Table 3, Table 4 and Figure 13.

Table 3. The Recognition Rates y Different Methods on ORL Face Database

\begin{tabular}{|c|c|c|c|c|c|c|c|c|c|}
\hline \multirow{2}{*}{ Methods } & \multicolumn{10}{|c|}{ Recognition Rate(\%) } \\
\cline { 2 - 10 } & 80 & 120 & 160 & 200 & 240 & 280 & 320 & 360 & ave \\
\hline Gabor & 82.06 & 86.23 & 92.31 & 90.50 & 94.37 & 95.57 & 96.25 & 95.89 & 91.64 \\
\hline LBP & 80.74 & 82.87 & 88.72 & 90.28 & 91.53 & 92.68 & 93.84 & 94.21 & 89.36 \\
\hline PCA & 78.83 & 82.54 & 83.67 & 88.10 & 89.68 & 92.30 & 92.18 & 92.87 & 87.52 \\
\hline $\begin{array}{c}\text { 2D- } \\
\text { Gabor+UPLBP }\end{array}$ & 86.67 & 90.41 & 92.81 & 95.65 & 97.67 & 97.36 & 97.82 & 98.73 & 94.63 \\
\hline
\end{tabular}

Table 4. The Used Time by Different Methods on ORL Face Database

\begin{tabular}{|c|c|c|c|c|c|c|c|c|}
\hline \multirow{2}{*}{ Methods } & \multicolumn{9}{|c|}{ Recognition Time(s) } \\
\cline { 2 - 9 } & 80 & 120 & 160 & 200 & 240 & 280 & 320 & 360 \\
\hline Gabor & 0.805 & 0.954 & 1.054 & 1.120 & 1.305 & 1.445 & 1.685 & 1.897 \\
\hline LBP & 0.560 & 0.640 & 0.755 & 0.874 & 0.983 & 1.124 & 1.315 & 1.653 \\
\hline PCA & 0.404 & 0.495 & 0.593 & 0.689 & 0.824 & 0.941 & 1.187 & 1.432 \\
\hline $\begin{array}{c}\text { 2D- } \\
\text { Gabor+UPLBP }\end{array}$ & 1.046 & 1.187 & 1.312 & 1.470 & 1.634 & 1.779 & 1.876 & 2.120 \\
\hline
\end{tabular}




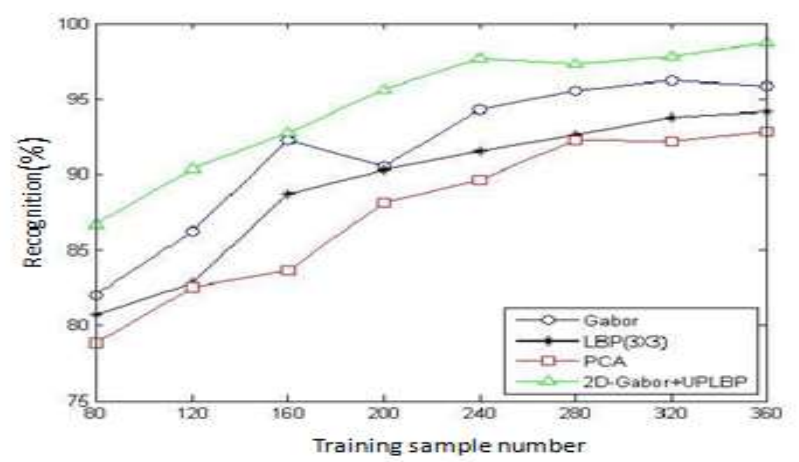

Figure 13. Comparison Chart of Recognition Rates for Four Methods on ORL Face Database

\section{Conclusion}

By comparing the experiments of three facial expression databases, the algorithm proposed by the thesis has a higher recognition rate than only using Gabor or LBP, as is shown by Table 3 and Fig.13. Furthermore, the average recognition rate of only using PCA is the lowest. Hence the thesis proposes the algorithm that combines the advantages of 2D-Gabor wavelet and LBP uniform mode: The 2D-Gabor wavelet abstracts the multidirection and multi-scale spatial frequency of local images. The LBP algorithm uniform mode represents the detailed information of the image zones near the image. In the meantime, the abstracted feature information is more comprehensive and effective. Hence the algorithm adopted by the thesis is better than the 2D-Gabor transform and LBP method. The method of abstracting characteristics only through PCA abstracts the overall characteristics of facial images from the overall profile of facial images. Hence it cannot describe the localized details and characteristics of facial images or represent the relations between regions. Moreover, some effective feature information is easily neglected. That's why the recognition rate is the lowest. As is shown by Table 4, the algorithm adopted by the thesis is highly complicated. Meanwhile, the time of identifying the single frame is longer than other three methods. If the pixel of inputting a facial image is $112 * 92,40$ images of the same size will be produced after the Gabor transform. Through the uniform pattern LBP (the division is $5^{*} 3$ ). In terms of recognition rate, it is more effective than other three feature abstraction algorithms. The correct recognition is the most crucial point in facial recognition. Hence the algorithm proposed by the thesis enjoys the highest feasibility.

\section{Acknowledgments}

This research was supported by the Busan Metropolitan City, Korea, under the 2017 BB21 program grants

\section{References}

[1] A. Fiat and A. Shamir, "How to prove yourself: practical solutions to identification and signature problem", Springer Berlin Heidelberg, (1986), pp. 186-164.

[2] R. W. Frischholz and U. Dieckmann, "BioID: a multimodal biometric identification system," Computer, vol. 33, no. 2, (2000), pp. 64-68.

[3] K. Uchida, "Frontiers of image processing technologies and applications. Biometrics personal identification and its application", Journal of the Institute of Electrical Engineers of Japan, vol. 120, no. 7, (2000), pp. 407-410.

[4] P. Wang and Q. Ji, "Multi-view face and eye detection using discriminant feature", Computer Vision \& Image Understanding, vol. 105, no. 2, (2005), pp. 99-111. 
[5] P. J. Phillips, P. J. Flynn, T. Scruggs, B. K. Bowyer, J. Chang, K. Hoffman, J. Marques, J. Min and W. Worek, "Overview of the face recognition grand challenge", IEEE Computer Society Conference on Computer Vision and Pattern Recognition, (2013), pp. 947-954.

[6] P.J. Phillips, P. Grother, R. Micheals, D.M. Blackburn, E. Tabassi and M. Bone, "Face Recognition Vendor Test 2002," IEEE International Workshop on Analysis and Modeling of Faces and Gestures, (2003), pp.44.

[7] R. Gottumukkal, "An improved face recognition technique based on modular PCA approach", Pattern Recognition Letters, vol. 25, no. 4, (2004), pp. 429-436.

[8] H. Zhang, B. Zhang, W. Huang and Q. Tian, "Gabor wavelet associative memory for face recognition", IEEE Transactions on Neural Networks, vol. 16, no. 1, (2005), pp. 275-278.

[9] W. Zhao, R. Chellappa, P. J. Phillips and A. Rosenfeld, "Face recognition: A literature survey", Acm Computer Surveys, vol. 35 , no. 4, (2003), pp. 399-458.

[10] A. Kumar and G. K. H. Pang, "Defect detection in textured materials using Gabor filters", IEEE Transactions on Systems Man \& Cybernetics Part B Cybernetics A Publication of the IEEE Systems Man \& Cybernetics Society, vol. 32, no. 5, (2002), pp. 553-570.

[11] X. Y. Tan and B. Triggs, "Enhanced local texture feature sets for face recognition under difficult lighting conditions", IEEE Transactions on Image Processing a Publication of the IEEE Signal Processing Society, vol. 19, no. 6, (2010), pp. 1635-1650.

[12] T. Ahonen, A. Hadid and M. Pietikainen, "Face description with local binary patterns: application to face recognition", IEEE Transactions on Pattern Analysis \& Machine Intelligence, vol. 28, no. 12, (2006), pp. 2037-2041.

[13] P. Duygulu, K. Barnard, J. F. G. Freitas and D. A. Forsyth, "Object recognition as machine translation: Learning a lexicon for a fixed image vocabulary", European Conference on Computer Vision, (2002), pp. 97-112.

[14] T. Ahonen, A. Hadid and M. Pietikainen, "Face description with local binary patterns: Application to face recognition", IEEE Transactions on Pattern Analysis and Machine Intelligence, vol. 28, no. 12, (2006), pp. 2037-2041.

[15] Z. H. Xie and S. Q. Wu, "Infrared face recognition using LBP and discrimination patterns", Journal of Image \& Graphics, vol. 17, no. 6, (2012), pp. 707-711.

[16] Q. Xu, J. Yang and S. Y. Ding, "Texture segmentation using LBP embedded region competition", Elcvia Electronic Letters on Computer Vision \& Image Analysis, vol. 5, no. 1, (2005), pp. 41-47.

[17] J. Zhang, C. J. Xie, L. T. Song, R. Li and H. B. Chen, "Robust image segmentation using LBP embedded region merging", TELKOMNIKA, vol. 14, no. 1, (2016), pp. 368-377.

[18] V. Bouffard and J. A. Ferland, "Improving simulated annealing with variable neighborhood search to solve the resource-constrained scheduling problem", Journal of Scheduling, vol. 10, no. 6, (2007), pp. 375-386.

\section{Authors}

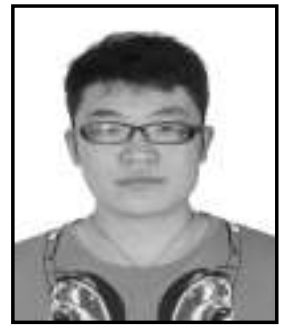

Jiyuan Wang, Wang Jiyuan received his B.S.at Tongmyong University in Korea(2010 2015). Currently, he is studying in Department of Information and Communications Engineering Tongmyong University, Korea for master degree. His main research areas are image processing and pattern recognition.

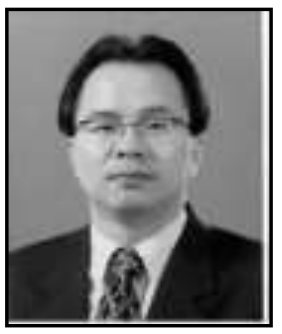

Eung-Joo Lee, Eung-Joo Lee received his B. S., M. S. and Ph. D. in Electronic Engineering from Kyungpook National University, Korea, in 1990, 1992, and Aug. 1996, respectively. Since 1997 he has been with the Department of Information \& Communications Engineering, Tongmyong University, Korea, where he is currently a professor. From 2000 to July 2002, he was a president of Digital Net Bank Inc. From 2005 to July 2006, he was a visiting professor in the Department of Computer and Information Engineering, Dalian Polytechnic University, China. His main research interests include biometrics, image processing, and computer vision. 
International Journal of Multimedia and Ubiquitous Engineering Vol.12, No.12 (2017) 